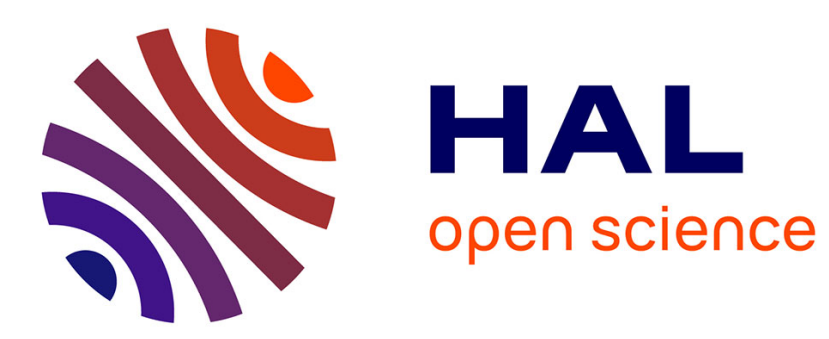

\title{
Event coreference and discourse relations
}

\author{
Laurence Danlos, Bertrand Gaiffe
}

\section{To cite this version:}

Laurence Danlos, Bertrand Gaiffe. Event coreference and discourse relations. L. Kulda. Language, Music and Cognition, Kluwer Academic Publishers, 2004. halshs-00082841

\section{HAL Id: halshs-00082841 https://shs.hal.science/halshs-00082841}

Submitted on 28 Jun 2006

HAL is a multi-disciplinary open access archive for the deposit and dissemination of scientific research documents, whether they are published or not. The documents may come from teaching and research institutions in France or abroad, or from public or private research centers.
L'archive ouverte pluridisciplinaire HAL, est destinée au dépôt et à la diffusion de documents scientifiques de niveau recherche, publiés ou non, émanant des établissements d'enseignement et de recherche français ou étrangers, des laboratoires publics ou privés. 


\title{
Event coreference and discourse relations
}

\author{
Laurence Danlos \\ LATTICE - TALANA \\ UFRL - Université Paris 7, \\ Case 7003 \\ 2, place Jussieu \\ 75251 Paris Cedex 05, France \\ danlos@linguist.jussieu.fr \\ Bertrand Gaiffe \\ LORIA - CNRS \\ Campus scientifique BP239 \\ 54506 Vandoeuvre lès Nancy Cedex, France \\ Bertrand.Gaiffe@loria.fr
}

December 18, 2002

\begin{abstract}
Event coreference is largely ignored in works on coreference as well as in works on temporal order in discourses, in which temporal relations between two eventualities $\mathrm{e}_{1}$ and $\mathrm{e}_{2}$ always suppose that $\mathrm{e}_{1} \neq$ $\mathrm{e}_{2}$. However, event coreference is not an uncommon phenomenon. We show that it is is the keystone on which at least four types of discourses are based: on the one hand, particularizing and generalizing discourses, which can be viewed as special cases of elaboration and reformulation discourses respectively, on the other hand causal discourses in which the effect is expressed by means of a causative verb, which are generally analyzed as explanation or result discourses. Descriptions and linguistic analyses of these discourses allow us to present unusual linguistic phenomena (e.g., coreference between existentially quantified elements, or assymetrical behavior of explanation or result discourses). The discourse relations involved are examined. This leads us to introduce and define new discourse relations. It also sheds a new light on the discourse relations Explanation and Result.
\end{abstract}




\section{Introduction}

Works on temporal relations between two eventualities $e_{1}$ and $e_{2}$ examine almost exclusively if one eventuality precedes, includes or overlaps with the other one [MS88, Ash93, Pus95]. All these temporal relations suppose that $\mathrm{e}_{1} \neq \mathrm{e}_{2}$. We will concentrate on cases where $\mathrm{e}_{1}=\mathrm{e}_{2}$, i.e., on event coreference. Event coreference can be seen as a particular case of either event elaboration or event reformulation, for which it has been acknowledged that time is not understood as moving [SC91].

Contrary to coreference between objects, coreference between events has hardly been studied in detail, except for a (pro)nominal phrase referring to an event as in (1) in which the pronoun this refers to the event described in the first sentence, see among others [Web88, Ash93].

(1) Ted arrived late. This irritated Mary.

Nevertheless, there is a number of other cases of event coreference since the description of an event e can be of three types: it is either a sentence, or a(pro)nominal phrase or an abstraction which is not linguistically realized. This last case occurs when $e$ is a sub-event of a complex event (cf. infra). Between two successive descriptions $\mathrm{D}_{1}$ and $\mathrm{D}_{2}$ of the same event $e$, there exist therefore $3^{2}=9$ cases of event coreference potentially observable depending on the types of $\mathrm{D}_{1}$ and $\mathrm{D}_{2}$. In this paper, the following cases are examined:

- In Section 2, event coreference when $\mathrm{D}_{1}$ and $\mathrm{D}_{2}$ are both sentences (noted as $\mathrm{S}_{i}$ ) as in $(2 \mathrm{a})$ and $(2 \mathrm{~b})$ is examined.

(2) a. The yugu team won a victory. They beat the tara team yesterday.

b. The yugu team beat the tara team yesterday. Therefore, they won a victory.

In (2a), the two sentences refer to the same event, with more information on that event in the second sentence (which "particularizes" the first one). In (2b), obtained from (2a) by reversing the order of the sentences, $\mathrm{S}_{2}$ "generalizes" $\mathrm{S}_{1}$.

- In Section 3, event coreference when $\mathrm{D}_{i}(\mathrm{i}=1,2)$ is a sentence and $\mathrm{D}_{j}(\mathrm{j}=1,2, \mathrm{j} \neq \mathrm{i})$ is not syntactically realized is examined. More precisely, causal discourses in which the effect is expressed by means 
of a causative verb (e.g., crack) are examined, as in (3a) and (3b) ((3b) is obtained from (3a) by reversing the order of the sentences).

(3) a. Ted cracked the carafe. He hit it against the sink.

b. Ted hit the carafe against the sink. He cracked it.

The causing sub-event of the complex event described by crack is not linguistically realized, it is just known that it is an act by Ted on the carafe. However, it is understood as coreferent to the event described in the cause sentence (Ted hit the carafe against the sink.).

Studying event coreference requires the definition of two types of coreference relation according to the quantity of information conveyed:

Two successive descriptions $D_{1}$ and $D_{2}$ of the same entity $x$ (event or object) are in a particularization relation noted as $D_{2}=P A R T\left(D_{1}\right)$ iff $D_{2}$ conveys some new information about $x$ when compared to the information known from $D_{1}$.

Two successive descriptions $D_{1}$ and $D_{2}$ of the same entity $x$ (event or object) are in a generalization relation noted as $D_{2}=G E N\left(D_{1}\right)$ iff $D_{2}$ does not bring any new information about $x$ (and $D_{1} \neq D_{2}$ ).

Let us illustrate these two types of coreference with the well-known case where $\mathrm{x}$ is an object. $\mathrm{D}_{1}$ and $\mathrm{D}_{2}$ are then (pro)nominal phrases. In (4a), the demonstrative NP this New Yorker girl = PART(an actress) since it brings new information (the actress is a New Yorker girl). In (4b), the pronoun she $=\operatorname{GEN}($ an actress): a pronoun cannot bring any new information. Finally, in (4c) the definite or demonstrative NP the / this artist $=\mathrm{GEN}$ (an actress) since artist, hyperonym of actress, does not bring new information.

(4) a. Ted had a drink with an actress. This New Yorker girl irritates Mary.

b. Ted had a drink with an actress. She irritates Mary.

c. Ted had a drink with an actress. The / This artist irritates Mary.

These two types of coreference relation according to the quantity of information conveyed do not play a major role in the literature on object coreference. However, they are essential for event coreference. In the next sections, it will be shown that (2a) and (3a) involve an event coreference relation of type particularization, (2b) and (3b) an event coreference relation of type generalization. This difference in the type of coreference explains assymetrical data. For example, it will be shown that any kind of adjunct can be inserted in the first sentence of $(2 \mathrm{a})$ or $(3 \mathrm{a})$, while the insertion of 
adjuncts in the first sentence of $(2 \mathrm{~b})$ or $(3 \mathrm{~b})$ is limited. As a consequence, the causal discourses in (3a) and (3b) are not really converse, despite what is generally believed. More generally, it will be shown that particularization and generalization are not symmetrical: the inverse of a generalization relation is a particularization relation, but the inverse of a particularization relation is not always a generalization relation.

We will show that it is necessary to lay down new discourse relations (the name of a discourse relation starts with a capital letter): Particularization (a particular case of Elaboration) for (2a) and Generalization (a particular case of Reformulation) for (2b). Of course, these new discourse relations are closely related to the types of event coreference: particularization (abbreviated as PART) for (2a) and generalization (abbreviated as GEN) for (2b). Moreover, we will show that these discourse relations are involved in causal discourses: Particularization for (3a) and Generalization for (3b). We will question the analysis of (3a) with Explanation and that of (3b) with Result, and shed a new light on these causal discourse relations. Discourse relations will be studied in the formal framework of SDRT (Segmented Discourse Representation Theory, [Ash93, AL95, AP00, ? $\left.]^{1}\right)$.

\section{Event coreference between two sentences}

Sections 2.1 and 2.2 provide linguistic descriptions, which are formalized in Section 2.3. Section 2.4 examines the discourse relations involved.

Notation: The symbol $\mathrm{X}_{i}^{r}$ with $\mathrm{i}=1$ or 2 denotes the predicate or an argument or modifier in $S_{i}$ with the role $\mathrm{r}$; for an argument $\mathrm{r}=$ agent, patient,... ; for a modifier $\mathrm{r}=$ time, location,... ; for the predicate, $\mathrm{r}$ is irrelevant.

\subsection{Particularizing discourses}

A particularization event coreference relation as defined above is to be found in "particularizing discourses" (henceforth PDs) such as (2a) or (5).

(5) Fred damaged a garment. He stained a shirt.

In (5), the same event is described in both sentences, with more information in $S_{2} . S_{2}$ particularizes $S_{1}$ since its predicate is a hyponym of the predicate

\footnotetext{
${ }^{1}$ We assume that the reader knows SDRT. If it is not the case, she can skip the SDRT formalizations presented here.
} 
in $S_{1}($ stain $<$ damage) and the (indefinite) object is a hyponym of the (indefinite) object in $S_{1}$ (shirt $<$ garment), while its subject is a (pronominal) anaphora of the subject in $S_{1}^{2}$.

More natural examples of PDs are observed when extralinguistic knowledge is at stake, as in (6). These PDs are based on an extension of hyperonymy, namely $X$ compliment $Y>X$ tell $Y$ that $Y$ is pretty in (6a), and $X$ be sick $>X$ have the flu in (6b). These extended hyperonymy relations rely on cultural or encyclopedic knowledge.

(6) a. Fred complimented Sue. He told her that she is pretty.

b. Fred is sick. He has the flu.

Our linguistic study is not corpus based. Nevertheless it is easy to find real examples of PDs, such as (7a) found in [Ash93, p. 1].

(7) a. (...) linguist and philosophers (..) developed a sophisticated understanding of events and states and the expressions that denote them. They developed a typology of such entities.

The particularization interpretation of a PD can be explicitly marked, as in (8) with the cue phrase more precisely.

(8) Fred damaged a garment. More precisely, he stained a shirt.

The PD in (5) or (8) gives rise to an unusual phenomenon: the two indefinite NPs a garment and a shirt are coreferent. This is unusual since it is generally admitted [KR93, Cor95] that an indefinite NP has an existential reading (i.e. introduces a new discourse referent) and that therefore two indefinite NPs are not coreferent ${ }^{3}$. This is the case in (9) in which two garments are involved, whereas only one garment is involved in (5). We will see in Section 2.4 how to compute the coreference of two indefinite NPs in PDs.

(9) Fred stained a garment. Joe tore a shirt.

\footnotetext{
${ }^{2}$ The two sentences are in the same tense, which is required for event coreference.

${ }^{3}$ A few cases exist in which two indefinite NPs are coreferent, (ia) and (ib) taken from [Cor94].

(i) a. A man arrived. He was a New Yorker.

b. A man, a New Yorker, arrived.

However, the examples presented here with two coreferent indefinite NPs have not been described in the literature, as far as we know.
} 
A discourse such as (5) in which at least one element in $S_{2}$ specifies a corresponding element in $S_{1}$ is called a "PD by specification". Another type of $\mathrm{PD}$ is given in (10). In (10), the modifier during dinner in $S_{2}$ provides temporal information about $e$; the other elements $\mathrm{X}_{2}^{r}$ in $S_{2}$ anaphorize or repeat the corresponding elements $\mathrm{X}_{1}^{r}$ in $S_{1}$.

(10) Fred stained a shirt. He stained it during dinner.

A discourse such as (10) in which at least one element $\mathrm{X}_{2}^{r}$ in $S_{2}$ has no corresponding element $\mathrm{X}_{1}^{r}$ in $S_{1}$ is called a "PD by adjunction". Specification and adjunction may be combined in a single PD. In (11), a shirt specifies a garment and the time adjunct in $S_{2}$ has no corresponding element in $S_{1}$.

(11) Fred stained a garment. He stained a shirt during dinner.

At face value, one gets a $\mathrm{PD}$ when each element $\mathrm{X}_{2}^{r}$ in $S_{2}$ is either an (extended) hyponym, an anaphora or a repetition of a corresponding element $\mathrm{X}_{1}^{r}$ in $S_{1}$ or has no corresponding element $\mathrm{X}_{1}^{r}$ in $S_{1}$.

To summarise, in text understanding, it has to be computed that there is only one garment and one event involved in $(5)^{4}$. In text generation, it must be determined under which conditions a PD may be uttered and which linguistic forms should be produced [Rou00].

\subsection{Generalizing restatement discourses}

A generalization event coreference relation as defined in Section 1 is to be found in "generalizing restatement discourses" (henceforth GDs) such as (12).

(12) a. Fred stained a shirt. Therefore, he damaged a garment.

b. Fred murdered Sue. Therefore, he committed a crime.

While a PD stands mainly at the informational level, a GD stands mainly at the intentional level: the first sentence in $(12 \mathrm{a})$ or $(12 \mathrm{~b})$ describes what the speaker wants the hearer to know about an event $e$, the second one consists in her restatement of $e$. By definition, a restatement cannot bring new information, but it can present an event in a new light, for example, if the speaker has the intention to forge links with other data, (13).

(13) Fred murdered Sue. Therefore, he committed a crime for which he risks the death sentence.

\footnotetext{
${ }^{4}$ See also [HGA97] for event coreference issues within an information extraction system.
} 
(12a) is created from (5) by reversing the order of the sentences (and the anaphoric relations), and by introducing a cue phrase like therefore, with an epistemic value [RJ96]. $S_{2}$ generalizes $S_{1}$ because damage and garment are respectively hyperonyms of the corresponding elements in $S_{1}$. In (12b), $S_{2}$ generalizes $S_{1}$ because the complex predicate commit a crime is a hyperonym of murder.

As for PDs, more natural examples of GDs are observed when an extended notion of hyperonymy is at stake, (14).

(14) a. Fred told Mary that she is pretty. Therefore, he complimented her. b. Fred has the flu. Therefore, he is sick.

At face value, one gets a GD when each element $\mathrm{X}_{2}^{r}$ in $S_{2}$ is an (extended) hyperonym, an anaphora or a repetition of a corresponding element $\mathrm{X}_{1}^{r}$ in $S_{1}$.

\subsection{Formalized conditions}

In [Dan01], the conditions to observe a PD or GD have been formalized in the framework of SDRT, using a notion of implication between two DRSs. Intuitively, in first approximation, in a $\mathrm{PD}, \mathrm{S}_{2}$ "implies" $\mathrm{S}_{1}$. Informally, staining a shirt "implies" damaging a garment, so Fred stained a shirt "implies" Fred damaged a garment in (5). At the opposite, in a GD, $\mathrm{S}_{1}$ "implies" $\mathrm{S}_{2}$.

Nevertheless, the situation is more complex: it is indeed the case that $S_{1}$ always "implies" $S_{2}$ in a GD. However, it is not always the case that $S_{2}$ "implies" $S_{1}$ in a PD. There is an assymetry between PDs and GDs. More precisely, if $S_{1}$. (therefore) $S_{2}$. is a GD, then $S_{2}$. $S_{1}$. is a PD. But the converse does not hold, as illustrated in (15). (15a) is a PD. $S_{1}$ in (15a) includes a time adjunct (during dinner) which is not repeated in $S_{2}$, although it is understood that the staining event occurred during dinner and that it is the same event as the damaging event. This means that the second sentence of a PD has to bring new information but has not to repeat all the information conveyed in the first sentence. On the other hand, the second sentence of a GD cannot bring new information at all. (15b), is incoherent (hence the sign \#) and cannot be interpreted as a GD.

(15) a. Ted damaged a garment during dinner. He stained a shirt.

b. \# Ted stained a shirt. Therefore, he damaged a garment during dinner. 
Another type of PD examples with no corresponding GD is illustrated in (16a): (16a) is a PD although the verb in $S_{2}$ is an hyperonym of the verb in $S_{1}$. It has no corresponding GD - (16b) is not a GD. (16a) is paraphrased by (16c) with the anaphoric form do it.

(16) a. Ted stained a shirt. He damaged the garment during dinner.

b. \# Ted damaged a shirt during dinner. Therefore, he stained it.

c. Ted stained a shirt. He did it during dinner.

To put it in a nutshell, the conditions to observe a GD (noted $\mathcal{C}_{G D}$ ) are based on a notion of implication between the DRSs which represent the sentences, while the conditions to observe a $\mathrm{PD}$ (noted $\mathcal{C}_{P D}$ ) are slightly more complex.

\subsection{Discourse relations}

It is likely that anyone working on discourse relations would lay down that the discourse relation between the two sentences of a PD is Elaboration. However, we are going to show that it is necessary to postulate the existence of a new discourse relation called "Particularization" which implies an event coreference. Beforehand, a terminological remark: "particularization" (abbreviated as PART) designates a type of coreference as defined in section 1; "Particularization" designates a discourse relation between two sentences. These two notions are linked in SDRT in the following way: if $\pi_{i}$ is the label for the DRS $K_{i}$ representing the sentence $S_{i}$, then $\mathrm{K}_{2}=\operatorname{PART}\left(\mathrm{K}_{1}\right) \Leftrightarrow$ $\operatorname{Particularization}\left(\pi_{1}, \pi_{2}\right)$.

Elaboration is given various definitions [Hob79, MT88, AL95], but whatever its definition, this discourse relation implies no event coreference relation. Let us illustrate this point with a "classic" example of Elaboration, (17a) or its shorter version, (17b). The first sentence denotes an event $\mathrm{e}_{1}$ which is "elaborated" in the next sentence(s) which bring(s) new information on one or some stages (sub-events) of $e_{1}$. No event coreference is at stake: a trip by plane cannot not be reduced to a takeoff and a landing and even less to a simple takeoff. There exists a number of stages in the trip which are described neither in (17a) nor in (17b).

(17) a. Nicholas flew from Austin to Paris. He took off at 6 am. He landed at $2 \mathrm{pm}$.

b. Nicholas flew from Austin to Paris. He took off at 6 am. 
Therefore it is necessary to postulate the existence of a discourse relation (Particularization) which implies an event coreference relation ${ }^{5}$. Only this discourse relation allows us to preserve well established insights on discourse referents. The argumentation is the following: examples such as (5) are productive counter-examples to the principle of the existential reading of an indefinite NP (which entails that two indefinite NPs are not coreferent). Hence, two solutions towards this principle are available:

- Given these productive counter-examples, this principle is abandoned and an anaphoric reading of indefinite NPs is proposed to allow the coreference of two indefinite NPs. This solution does not seem sound since this principle is nearly always true, except in PDs (and in GDs and in some well known exceptions, see note 2).

- This principle is not abandoned. The two indefinite NPs in (5) are represented in the standard way: two discourse referents $\mathrm{x}$ and $\mathrm{y}$ with the complete conditions garment(x) and shirt(y) are introduced. Yet the coreference relation $\mathrm{x}=\mathrm{y}$ is established thanks to the discourse relation Particularization. This discourse relation implies an event coreference relation which implies in turn that the arguments with the same role are coreferent. In other words, it is because the discourse relation Particularization is established between the two sentences of (5) that it is known that the two sentences refer to the same event, and therefore that the two patients a garment and a shirt are coreferent, i.e., $\mathrm{x}=\mathrm{y}$.

This last solution seems better. It raises the following question: how can it be established that the discourse relation in (5) is Particularization (which amounts to establishing that (5) is a PD)? The answer to this question lies in linguistic knowledge: the two sentences in a PD follow some strong linguistic constraints which have been described in Sections 2.1 and 2.3. This set of constraints, noted as $\mathcal{C}_{P D}$, allows the computation of the discourse relation Particularization. In SDRT, the rule is the following (where $>$ is the symbol for the default implication): $\left(\tau, \pi_{1}, \pi_{2}\right) \wedge \mathcal{C}_{P D}>\operatorname{Particularization}\left(\pi_{1}, \pi_{2}\right)$.

\section{Summary on event coreference between two sentences}

It is necessary to postulate the existence of a new discourse relation, Particularization, which implies an event coreference relation between the

\footnotetext{
${ }^{5}$ In the framework of SDRT, the indefeasable consequence of Particularization is written in the following way: Particularization $\left(\pi_{1}, \pi_{2}\right) \rightarrow \operatorname{Main}-\operatorname{event}\left(\pi_{1}\right)=\operatorname{Main}-\operatorname{event}\left(\pi_{2}\right)$.
} 
event $\mathrm{e}_{1}$ and $\mathrm{e}_{2}$ described in the first and second sentences. It allows the establishment of an event coreference relation although the events involved are existentially quantified. This event coreference relation implies the coreference of participants with the same thematic role, even if they are existentially quantified. This discourse relation differs from Elaboration for which only one or some stages (sub-events) of $\mathrm{e}_{1}$ are specified. Nevertheless, Particularization can be seen as a particular case of Elaboration. For exactly the same reason, a discourse relation Generalization has to be laid down in order to establish the event co-reference in such examples as (12).

\section{Causal discourses}

\subsection{Linguistic description}

Examples (3a) et (3b), repeated in (18a) and (18b), express a "direct causation" [Fod70, Sch75]. It is generally admitted that the discourse relation is Explanation in (18a) and Result in (18b). We adopt this position in this section before questioning it in the next section.

(18) a. Ted cracked the carafe. He hit it against the sink.

b. Ted hit the carafe against the sink. He cracked it.

In these causal discourses, the effect is expressed by means of a causative verb, here crack. It is well known (see among other [MS88, Pus91, Pus95]) that a causative verb denotes a complex event which can be broken down into:

- a causing sub-event, here a non specified $^{6}$ act by Ted on the carafe which is represented in the simplified ${ }^{7}$ DRS $\pi_{1}$ : $\mathrm{e}_{1}-$ ?ACT $(\mathrm{t}, \mathrm{c})$,

- and a resulting state, here the cracked state of the carafe which is represented by $\pi_{2}: \mathrm{e}_{2}-\operatorname{cracked}(\mathrm{c})$.

$\pi_{1}$ and $\pi_{2}$ are grouped in the DRS $\pi_{3}$, which is noted as $\pi_{3}=\left(\pi_{1}, \pi_{2}\right)$. $\pi_{3}$ can be viewed as the DRS representing the sentence with the causative verb (Ted cracked the carafe.). The analysis of a clause with a causative verb is sketched in Figure 1.

\footnotetext{
${ }^{6}$ The act by Ted on the carafe is not specified, however it is understood as an act which affects its patient, i.e., the carafe.

${ }^{7}$ Our simplified graphic representation of DRS includes conditions (in a compact way) but not the universe. We leave it to the reader to re-establish the complete DRSs.
} 


$$
\begin{aligned}
& \text { Ted cracked the carafe. } \\
& \begin{array}{c}
\pi_{1}=\mathrm{e}_{1}-? \mathrm{ACT}(\mathrm{t}, \mathrm{c}) \\
\pi_{2}=\mathrm{e}_{2}-\operatorname{cracked}(\mathrm{c}) \\
\pi_{3}=\left(\pi_{1}, \pi_{2}\right)
\end{array}
\end{aligned}
$$

Figure 1: Analysis of a clause with a causative verb

The causal interpretation of discourses such as (18a) or (18b) can be explained in linguistic terms thanks to the notion of event coreference.

The cause sentence denotes an event which is interpreted as coreferent to the causing sub-event of the complex event referred to by the causative verb.

For example, in (18a) or (18b), the hitting event is coreferent to the causing sub-event of crack, i.e. the non specified act by Ted on the carafe. Let us underline that the causal interpretation of the discourses we are concerned with is totally bound to event coreference. To emphasize this point, consider the discourses in (19). Normally they should not be given a causal interpretation. However, if the hearer imagines that Ted is James Bond equipped with a special camera, then she may assume a causal interpretation of (19). In such a case, she assumes an event coreference between the taking a photo event ${ }^{8}$ and the causing sub-event of crack.

(19) a. Ted cracked the carafe. He took a photo of it.

b. Ted took a photo of the carafe. He cracked it.

The analysis of (18a) is schematized in Figure 2 with the event coreference relation $\mathrm{e}_{1}=\mathrm{e}_{4}$, where $\mathrm{e}_{4}$ is the hitting event. $\pi_{1}$ introduces an underspecified predicate ?-ACT. This predicate is specified coreferentially in $\pi_{4}$ which labels $\mathrm{K}_{4}$, the DRS for the hitting sentence. hit can be considered as an hyponym of ?-ACT. The event coreference relation $\mathrm{e}_{1}=\mathrm{e}_{4}$ is therefore of type particularization, i.e. $\mathrm{K}_{4}=\operatorname{PART}\left(\mathrm{K}_{1}\right)$, since $\mathrm{K}_{4}$ brings new information as compared to $K_{1}$. Thus, Particularization $\left(\pi_{1}, \pi_{4}\right)$ can be laid down.

\footnotetext{
${ }^{8}$ The taking a photo event is then understood as an act which affects its patient, see note 6 .
} 


$$
\begin{aligned}
& \text { Ted cracked the carafe. He hit it against the sink. } \\
& \qquad \begin{aligned}
& \pi_{1}= \mathrm{e}_{1}-\text { ?ACT }(\mathrm{t}, \mathrm{c}) \\
& \pi_{2}= \mathrm{e}_{2}-\operatorname{cracked}(\mathrm{c}) \\
& \pi_{3}=\left(\pi_{1}, \pi_{2}\right) \\
& \pi_{4}= \mathrm{e}_{4}-\mathrm{hit}(\mathrm{t}, \mathrm{c}, \mathrm{s}) \\
& \mathrm{K}_{4}=\operatorname{PART}\left(\mathrm{K}_{1}\right) \Leftrightarrow \text { Particularization }\left(\pi_{1}, \pi_{4}\right) \\
& \text { with } \mathrm{e}_{1}=\mathrm{e}_{4}
\end{aligned}
\end{aligned}
$$

Fig.2 : Analysis of (18a) (preliminary version)
Ted hit the carafe against the sink. He cracked it.

$$
\begin{aligned}
\pi_{5} & =\mathrm{e}_{5}-\operatorname{hit}(\mathrm{t}, \mathrm{c}, \mathrm{s}) \\
\pi_{6} & =\mathrm{e}_{6}-? \mathrm{ACT}(\mathrm{t}, \mathrm{c}) \\
\pi_{7} & =\mathrm{e}_{7}-\operatorname{cracked}(\mathrm{c}) \\
& \pi_{8}=\left(\pi_{6}, \pi_{7}\right)
\end{aligned}
$$

$$
\mathrm{K}_{6}=\operatorname{GEN}\left(\mathrm{K}_{5}\right) \Leftrightarrow \text { Generalization }\left(\pi_{5}, \pi_{6}\right)
$$

$$
\text { with } \mathrm{e}_{5}=\mathrm{e}_{6}
$$

Fig.3 : Analysis of (18b) (preliminary version)

The analysis of $(18 \mathrm{~b})$, similar to that of $(18 \mathrm{a})$, is schematized in Figure 3 with the event coreference relation $\mathrm{e}_{5}=\mathrm{e}_{6}{ }^{9}$. This coreference relation is of type generalization, i.e. $\pi_{6}=\operatorname{GEN}\left(\pi_{5}\right)$, since $\mathrm{K}_{6}$ does not bring new information compared to $\mathrm{K}_{5}$. $\mathrm{K}_{6}$ can be viewed as an anaphora by hyperonymy of $\mathrm{K}_{5}$.

The undebatable link between causal interpretation and event coreference is shared by both Explanation and Result discourses. However these discourses differ in that Explanation discourses rely on an event coreference of type particularization, whereas Result discourses rely on a generalization event coreference. In parallel with the fact that some PDs do not have a corresponding GD (Section 2.3), some Explanation discourses do not have a corresponding Result discourse. Let us illustrate this point with the adjunction of modifiers in the sentence with a causative verb ${ }^{10}$. Two types of adjuncts must be distinguished:

- those with scope over the causing sub-event, for example, temporal adverbs (during dinner). In Ted cracked the carafe during dinner, during dinner has scope over the the causing sub-event. The information known on this event is: $e_{1}-? A C T(t, c) \wedge$ during-dinner $\left(e_{1}\right)$.

- those without scope over the causing sub-event, for example "quantifier" adverbs (badly). In Ted cracked the carafe badly, badly has scope over the resulting state but not over the causing sub-event. The information on the causing sub-event is $e_{1}-? A C T(t, c)$, and that on the resulting state is $e_{2}-\operatorname{cracked}(c) \wedge \operatorname{badly}\left(e_{2}\right)$.

\footnotetext{
${ }^{9}$ The numbering $\pi_{5}-\pi_{8}$ is used instead of the standard one $\pi_{1}-\pi_{4}$ to refer later to the DRSs in figures 1 and 2 without ambiguity.

${ }^{10}$ The claim that Explanation discourses rely on an event coreference of type particularization, whereas Result discourses rely on a generalization event coreference is thoroughly justified in [Dan00].
} 
The adjunction (in the sentence with a causative verb) of a modifier with scope over the causing sub-event, e.g., temporal adverb, does not alter the causal interpretation when Explanation is involved, see (20a) with the adjunction of during dinner in the crack sentence. But it does alter it when Result is involved, see (20b) which is marked with the sign \# to indicate that no causal interpretation is at stake ${ }^{11}$. This assymetry can be explained by a principle on the type of the event coreference relation: it has to be of type particularization for Explanation, as it is in (20a), see (21a) which resumes the information known from (20a). It has to be of type generalization for Result, which is not the case in (20b), see (21b), which resumes the information known from (20b) and where the generalization coreference relation is negated, which goes along with the fact that (20b) has no causal interpretation.

(20) a. Ted cracked the carafe during dinner. He hit it against the sink.

b. \# Ted hit the carafe against the sink. He cracked it during dinner.

(21) a. $e_{4}-h i t(t, c, s)=P A R T\left(e_{1}-? A C T(t, c) \wedge\right.$ during-dinner $\left.\left(e_{1}\right)\right)$ with $\mathrm{e}_{1}=\mathrm{e}_{4}$

b. $\neg\left[e_{6}-? A C T(t, c) \wedge\right.$ during-dinner $\left.\left(e_{6}\right)=G E N\left(e_{5}-h i t(t, c, s)\right)\right]$ with $\mathrm{e}_{5}=\mathrm{e}_{6}$

On the other hand, the adjunction of a of a modifier without scope over the causing sub-event,e.g., "quantifier" adverb, is possible in both Explanation and Result discourses while maintening a causal interpretation, (22). This goes along with the fact that the coreference relation is of type particularization in (22a), see (23a), and of type generalization in (22b), see (23b).

(22) a. Ted cracked the carafe badly. He hit it against the sink.

b. Ted hit the carafe against the sink. He cracked it badly.

(23) a. $e_{4}-h i t(t, c, s)=P A R T\left(e_{1}-? A C T(t, c)\right)$ with $\mathrm{e}_{1}=\mathrm{e}_{4}$

b. $e_{6}-? A C T(t, c)=G E N\left(e_{5}-h i t(t, c, s)\right)$ with $\mathrm{e}_{5}=\mathrm{e}_{6}$

Let us move to psychological causative verbs (e.g. irritate) whose subject can refer to an event (This incident irritated Mary) contrarily to causative verbs which denote a physical change of state $(*$ This incident cracked the carafe). Two types of discourses are thus available to express Result when

\footnotetext{
${ }^{11}$ As shown by the fact that it is impossible to insert a causal connective such as therefore between the two sentences.
} 
the change of state is psychological, as illustrated in (24): the subject of irritate refers to the cause in (24a) and to the agent of the cause in (24b). Our analysis puts forward that these two discourses, which describe the same situation but differs in communicative structure, involve both an event coreference relation. This coreference relation is "classic" in (24a) : (pro)nominal NP referring to an event. In (24b), it holds between the event referred to in the cause sentence and the causing sub-event of the causative verb. It can be considered that (24b) involves a metonymy: he is used instead of his act. With a psychological verb, Result can therefore be expressed by the metonymic or full form. With a causative non-psychological verb, only the metonymic form is available.

(24) a. Ted hit the carafe against the sink. This / This incident irritated Mary.

b. Ted hit the carafe against the sink. He irritated Mary.

\subsection{Discourse relations}

We are going to shed a new light on Explanation and Result. It is common knowledge to state that these discourse relations apply when a causal relation is involved, and when, for Explanation, the description of the effect precedes that of the cause, while for Result, the inverse discursive linear order is observed. These discourse relations can thus be established only when the following axioms (necessary conditions) are verified ${ }^{12}$ :

A1 : Explanation and Result hold between two DRSs, one representing the cause, the other one the effect.

A2 : It is possible to associate with these two DRSs two distinct syntactic realizations (which are thus linearly ordered); if the syntactic realization associated with the effect DRS precedes that associated with the cause DRS, Explanation applies; with the inverse discursive order, Result applies.

We are going to show that two opposite discourse theories, RST (Rhetorical Structure Theory, [MT88]) and SDRT in its [AP00]) version (noted as

\footnotetext{
${ }^{12}$ These axioms are written in SDRT style, however they do not constitute SDRT rules to infer Explanation or Result. More generally, in this section, the issue is to determine which discourse relations should be established in discourses such as (18a) or (18b). The issue is not to compute these discourse relations.
} 
SDRT-00), lay down Explanation or Result in such ways that A1 is not verified for RST while A2 is not verified for SDRT-00. Next we will propose a solution such that both $\mathrm{A} 1$ and $\mathrm{A} 2$ are verified.

In RST, discourse relations hold only between two sentences or two clauses (or between two DRSs representing sentences or clauses in the SDRT framework). As a consequence, it is said that Explanation holds between the two sentences of (18a) and Result between the two sentences of (18b). This amounts to Explanation $\left(\pi_{3}, \pi_{4}\right)$ and Result $\left(\pi_{5}, \pi_{8}\right)$ in the DRSs of figures 1 and 2. However this analysis is inappropriate: the sentence with the causative verb refers both to the cause (it indicates who is the agent) and to the effect. Axiom A1 is thus not verified.

Contrarily to RST, in SDRT-00, discourse relations hold between two DRSs without any constraint on their possible syntactic realizations. Therefore, Asher and Pustejovsky can propose to record in the lexical entry of a causative verb that the DRSs for the causing sub-event and the resulting state are linked by Result. This amounts to $\operatorname{Result}\left(\pi_{1}, \pi_{2}\right)$ and $\operatorname{Result}\left(\pi_{6}, \pi_{7}\right)$ in our examples. However, there is no criterion to choose Result rather than Explanation: the DRSs of the two sub-events involved are only abstract representations without corresponding syntactic realizations. Axiom A2 is thus not verified. The choice of Result rather than Explanation can be explained by the fact that Asher and Pustejovsky chose the discourse relation which follows the chronological order of the events (the cause precedes the effect). However, it is necessary to maintain a distinction between discursive and temporal orders. Recall that the discourses (18a) and (18b), with the same temporal order and different discursive orders, are based either on a particularization or generalization event coreference, which implies assymetrical behavior, e.g., for the insertion of adjuncts.

It is thus not justified to link by Result the DRSs for the causing subevent and the resulting state of a causative verb. On the other hand, we propose to link these two DRSs by means of the events they represent: we propose a conceptual (non discursive) link, noted as CAUSE, between the events involved, e.g. $\operatorname{CAUSE}\left(\mathrm{e}_{1}, \mathrm{e}_{2}\right)$ and $\operatorname{CAUSE}\left(\mathrm{e}_{6}, \mathrm{e}_{7}\right)$ in our examples. This link can be recorded in the lexical entry of a causative verb.

We propose moreover to lay down that the DRSs for the cause sentence and the resulting state are linked by Explanation or Result, e.g. Explanation $\left(\pi_{2}, \pi_{4}\right)$ and Result $\left(\pi_{5}, \pi_{7}\right)$ in our examples. Let us show that both axioms A1 and A2 are verified with this solution; the argumentation will be presented only for Explanation $\left(\pi_{2}, \pi_{4}\right)$, since that for Result $\left(\pi_{5}, \pi_{7}\right)$ is entirely similar. 
Ted cracked the carafe. He hit it against the sink.

$$
\begin{aligned}
& \pi_{1}=\mathrm{e}_{1}-? \operatorname{ACT}(\mathrm{t}, \mathrm{c}) \\
& \pi_{2}=\mathrm{e}_{2}-\operatorname{cracked}(\mathrm{c}) \\
& \pi_{3}=\left(\pi_{1}, \pi_{2}\right) \text { with } \operatorname{CAUSE}\left(\mathrm{e}_{1}, \mathrm{e}_{2}\right) \\
& \pi_{4}=\mathrm{e}_{4}-\operatorname{hit}(\mathrm{t}, \mathrm{c}, \mathrm{s})
\end{aligned}
$$

$\operatorname{Particularization}\left(\pi_{1}, \pi_{4}\right) \rightarrow \mathrm{e}_{1}=\mathrm{e}_{4}$ Explanation $\left(\pi_{2}, \pi_{4}\right)$

Fig.4 : Analysis of (18a)
Ted hit the carafe against the sink. He cracked it.

$$
\begin{gathered}
\pi_{5}=\mathrm{e}_{5}-\operatorname{hit}(\mathrm{t}, \mathrm{c}, \mathrm{s}) \\
\pi_{6}=\mathrm{e}_{6}-? \operatorname{ACT}(\mathrm{t}, \mathrm{c}) \\
\pi_{7}=\mathrm{e}_{7}-\operatorname{cracked}(\mathrm{c}) \\
\pi_{8}=\left(\pi_{6}, \pi_{7}\right) \text { with } \operatorname{CAUSE}\left(\mathrm{e}_{6}, \mathrm{e}_{7}\right) \\
\text { Generalization }\left(\pi_{5}, \pi_{6}\right) \rightarrow \mathrm{e}_{5}=\mathrm{e}_{6} \\
\operatorname{Result}\left(\pi_{5}, \pi_{7}\right)
\end{gathered}
$$

Fig.5 : Analysis of (18b)

- For the axiom A1 in Explanation $\left(\pi_{2}, \pi_{4}\right)$, it has to be verified that $\pi_{4}$ represents the cause, and $\pi_{2}$ the effect. This is the case: these DRSs describe the events $\mathrm{e}_{4}$ and $\mathrm{e}_{2}$ which are linked by the conceptual link CAUSE thanks to the following implication: $\operatorname{CAUSE}\left(\mathrm{e}_{1}, \mathrm{e}_{2}\right) \wedge \mathrm{e}_{1}=$ $\mathrm{e}_{4} \Rightarrow \operatorname{CAUSE}\left(\mathrm{e}_{4}, \mathrm{e}_{2}\right)$.

Note that a formal basis for A1 can be given, see A1'.

\section{A1': Explanation and Result hold between two DRSs which describe two events linked by CAUSE.}

- For the axiom A2 in Explanation $\left(\pi_{2}, \pi_{4}\right)$, it has to be verified that the syntactic realization associated with the effect DRS, i.e., $\pi_{2}$, precedes that associated with the cause DRS, i.e., $\pi_{4}$. This is the case: $\pi_{2}$ has no syntactic realization, however, $\pi_{2}$ is an element of $\pi_{3}$ which does have a syntactic realization, namely the sentence with the causative verb. This sentence precedes the syntactic realization of $\pi_{4}$, the cause sentence. Thereby, by associating with $\pi_{2}$, element of $\pi_{3}$, the syntactic realization of $\pi_{3}$, A2 is verified for Explanation $\left(\pi_{2}, \pi_{4}\right)^{13}$.

To sum up, the only way to lay down that (18a) involves Explanation and (18b) Result while having axioms A1 and A2 both verified consists in stating that these discourse relations link the DRSs for the cause sentence and the resulting state. The analyses of (18a) and (18b) which take into account these facts are sketched in figures 4 and 5 .

\section{Summary on causal discourses}

\footnotetext{
${ }^{13}$ The same kind of argumentation does not work for Result $\left(\pi_{1}, \pi_{2}\right)$ as proposed by Asher and Pustejovsky, since $\pi_{1}$ et $\pi_{2}$ are both elements of $\pi_{3}$ : the only syntactic realizations that can be associated with $\pi_{1}$ and $\pi_{2}$ are not distinct thus not linearly ordered.
} 
We have shown that the causal interpretation of discourses such as (18a) and $(18 \mathrm{~b})$ is bound to event coreference. Therefore, the discourse relations Particularization and Generalization are also at stake in causal discourses. What is mere Explanation and Result hold neither between the two sentences of the causal discourses nor between the two sub-events of the causative verb. They hold between the DRSs for the cause sentence and the resulting state. The fact that Explanation goes along with Particularization while Result goes along with Generalization allows us to explain why Explanation discourses have a normal behaviour (e.g. any modifier can be inserted) while Result discourses have an awkward behaviour (e.g. heavy constraints on the insertion of modifiers). Explanation and Result are thus not really converse, despite what is generally believed.

\section{Conclusion}

\section{References}

[AL95] N. Asher and A. Lascarides. Lexical disambiguation in a discourse context. Journal of Semantics, 12(12):69-108, 1995.

[AP00] N. Asher and J. Pustejovsky. The metaphysics of words in context. Journal of Logic, Language and Information, 2000.

[Ash93] N. Asher. Reference to Abstract Objects in Discourse. Kluwer, Dordrecht, 1993.

[Cor94] F. Corblin. La condition de nouveaut comme dfaut. Faits de langues (Presses Universitaires de France), (4):147-160, 1994.

[Cor95] F. Corblin. Les formes de reprise dans le discours, Anaphores et chanes de rfrence. Presses Universitaires de Rennes, Rennes, 1995.

[Dan00] L. Danlos. Event coreference in causal discourses. In P. Bouillon and F. Busa, editors, Meaning of Word. Cambridge University Press., 2000.

[Dan01] L. Danlos. Event coreference between two sentences. In H. Bunt, editor, Computing Meaning (volume 2). Kluwer Academic Publishers, Amsterdam, 2001.

[Fod70] J. Fodor. Three reasons for not deriving kill from cause to die. Linguistic Inquiry, 1970. 
[HGA97] K. Humphreys, R. Gaizauskas, and S. Azzam. Event coreference for information extraction. In Proceedings of the Workshop on Operational Factors in Pratical, Robust Anaphora Resolution for Unrestricted Texts, 35th Meeting of ACL, Madrid, 1997.

[Hob79] J. Hobbs. Coherence and coreference. Cognitive Science, (3):6790, 1979.

[KR93] H. Kamp and U. Reyle. From Discourse to Logic. Kluwer Academic Publishers, Dordrecht, 1993.

[MS88] M Moens and M. Steedman. Temporal ontology and temporal reference. Computational Linguistics, 14:15-28, 1988.

[MT88] W. Mann and S. Thomson. Rhetorical structure theory: Toward a functional theory of text organization. Text: An Interdisciplinary Journal for the Study of Text, 8(2), 1988.

[Pus91] J. Pustejovsky. The syntax of event structure. In Lexical and Conceptual Semantics. Elsevier Science Publishers, Amsterdam, 1991.

[Pus95] J. Pustejovsky. The generative Lexicon. The MIT Press, 1995.

[RJ96] C. Rossari and J. Jayez. Donc et les consécutifs ; des systèmes de contraintes référentielles. Lingvisticae Investagiones, 1996.

[Rou00] L. Roussarie. Un modle théorique d'inférences de structures sémantiques et discursives dans le cadre de la génération automatique de textes. Thèse de doctorat en linguistique, Université Denis Diderot, Paris 7, 2000.

[SC91] B. Spejewski and G. Carlson. Reference time relations. In The Ohio State University Press, editor, Proccedings from the Eastern States Conference on Linguistics, Baltimore, 1991.

[Sch75] R. Schank. Conceptual Information Processing. Amsterdam:North Holland, 1975.

[Web88] B. Webber. Discourse deixis: Reference to discourse segments. In Proceedings of ACL'1988, pages 113-123, Buffalo, NY, 1988. 